\title{
Monitoramento das ações pró-redução da transmissão vertical da sífilis na rede pública do Distrito Federal
}

Leonor Henriette de Lannoy Coimbra Tavares ${ }^{1}$

Onã Silva²

Recebido em: 20/08/2011

Aceito em: 14/02/2012

Leidijany Costa Paz ${ }^{3}$

Luiz Antônio Bueno Lopes ${ }^{4}$

Maria Liz Cunha de Oliveira ${ }^{5}$

Maria Marta Lopes Macedo ${ }^{6}$

Sonia Geraldes ${ }^{7}$

A sífilis é um agravo de grande preocupação epidemiológica e tem critérios para cumprir a meta de sua eliminação. Objetivou-se, nesta pesquisa, a apresentação do perfil epidemiológico de gestantes e parturientes atendidas na rede pública do Distrito Federal. Analisaram-se informações sobre a transmissão vertical da sífilis em 3.726 mulheres participantes. Coletaram-se dados primários e secundários utilizando a entrevista, informações de cartão de pré-natal e prontuários. Nos resultados, observou-se que muitas gestantes não foram testadas no pré-natal, segundo o protocolo de três testes de sífilis, diretriz expressa na portaria distrital no 37/2008. Detectaram-se desigualdades de testagem em mulheres negras, adolescentes e com baixa escolaridade e renda per capita. Conclui-se que, na atenção básica, exige o cumprimento de protocolos instituídos para a sífilis.

Descritores: Gestantes, Parturientes, Transmissão Vertical da Síflis, Epidemiologia.

\section{Syphilis is a health problem of great epidemiological concern and has criteria to meet the goal of its elimination.}

The aim of this research is the presentation of the epidemiological profile of pregnant women and pregnant women assisted in the public network in the Federal District. It was analyzed information about the transmission of syphilis in 3726 women participants. Data were collected using primary and secondary interviews, information card and prenatal records. The results showed that many pregnant women have not been tested during prenatal care, according to the protocol of three tests for syphilis, district guidelines expressed in the decree No. 37/2008. Inequalities were detected testing for black women, adolescents with low education and income per capita. We conclude that, in primary care requires compliance with established protocols for syphilis.

Descriptors: Pregnancy, childbirth, vertical transmission of syphilis, Epidemiology.

\section{Seguimiento de las acciones pro la reducción de la transmisión vertical de la sífilis en público en el Distrito Federal}

La sífilis es un agravio que preocupa y tiene criterios epidemiológicos para cumplir con la meta de eliminación. El objetivo de esta investigación es la presentación del perfil epidemiológico de las mujeres embarazadas y las mujeres embarazadas observados en público en el Distrito Federal. Se analizó información sobre la transmisión de la sífilis en 3726 mujeres participantes. Los datos primarios y secundarios fueron recolectados a través de entrevista, informaciones de tarjeta de prenatal y registros. Los resultados mostraron que muchas mujeres embarazadas no han sido probadas durante la atención prenatal, según el protocolo de tres pruebas para sífilis, normas expresas en el decreto No. 37/2008. Desigualdades de pruebas fueron detectadas en mujeres negras, jóvenes con baja escolaridad y renta per cápita. Se concluye que en la atención básica es necesario el cumplimiento de protocolos establecidos para la sífilis.

Descriptores: Embarazo, parto, la transmisión vertical de la sífilis, epidemiología.

1 Enfermeira, mestre em ciências da saúde, gerente da Unimista Sul/SES-DF-DF. E-mail: delannoytavares@gmail.com.

2 Enfermeira, especialista em saúde pública, mestre em educação, doutoranda no programa de pós-graduação em enfermagem da UnB, docente da Escola Superior de Ciências da Saúde (ESCS/SES-DF).

3 Enfermeira, mestre em avaliação de programas e processos em saúde, atua na área de vigilância epidemiológica - Gerência de DST e Aids/Divep/SVS/SES.

4 Médico, mestre em efetividade em saúde baseada em evidências, atua na área de análise de informação em saúde - Diretoria em Vigilância Epidemiológica/SVS/SES-DF. 5 Enfermeira, doutora em ciências da saúde, docente da Universidade Católica de Brasília, atua na área de transmissão vertical da síflis - Gerência de DST e Aids/Divep/SES. 6 Enfermeira, especialista em planejamento em saúde, atua em Unidade de Atenção Básica na SES-DF.

7 Médica, mestre em informação em saúde, atua na diretoria de vigilância epidemiológica da Subsecretaria de Vigilância Epidemiológica (Divep/SES-DF). 


\section{INTRODUÇÃO}

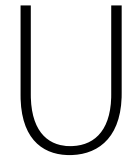

ma indagação recorrente acontece na epidemiologia brasileira: por que a sífilis continua sendo um grande problema de saúde pública? Das várias doenças transmitidas durante o ciclo grávido-puerperal, por que a síflis é a que tem as maiores taxas de transmissão?

O perfil epidemiológico dos agravos - sífilis em gestantes e congênita - resultante dos casos notificados e investigados gera tais indagações e justifica constantes estudos. Por exemplo, no Distrito Federal, a despeito do declínio da sífilis congênita - de 3,4 casos por mil NV a 1,6 por mil NV, de 2004 a 2009 - em 2010, observou-se um recrudescimento quanto à incidência da transmissão vertical da sífilis, que subiu para 2,1 casos por mil $\mathrm{NV}^{(1)}$.

Também nos resultados de estudos de natureza epidemiológica emergem problemas relativos à implementação das políticas pró-redução da TV do HIV e sífilis, falhas no diagnóstico, tratamento e ações de vigilância epidemiológica, entre outros.

Conforme diretrizes nacionais para a redução das taxas de sífilis congênita, destaca-se o protocolo da solicitação de testes não treponêmicos (VDRL) nos seguintes estágios: primeiro e terceiro trimestres gestacionais e no momento do parto. Tal recomendação, feita pela Organização Mundial da Saúde (OMS), foi acatada pelo Ministério da Saúde brasileiro, sendo a sífilis congênita considerada "indicador de avaliação da atenção básica à saúde" aos gestores municipais do Sistema Único de Saúde ${ }^{(2-4)}$. No Distrito Federal, preconizou-se a solicitação de três testes de sífilis (VDRL) durante a gravidez - um em cada trimestre gestacional - e o quarto teste no momento do parto(5).

Em 2007, a instância superior - Ministério da Saúde (MS) -, baseando-se em dados epidemiológicos dos referidos agravos, lançou o Plano Nacional de Redução da Transmissão Vertical (TV) do HIV e da sífilis. Definiramse responsabilidades e metas de redução regionalizada e gradativa das taxas desses agravos ${ }^{(4,6)}$. No mesmo ano, a Gerência de DST e Aids e Hepatites Virais (GEDST) do Distrito Federal realizou uma experiência de pactuação de ações pró-redução da TV do HIV e sífilis, resultando na portaria $n^{\circ} 37 / 2008^{(5,7)}$.

Posteriormente, a GEDST aderiu à pesquisa "Monitoramento das Informações Relacionadas à Realização dos Testes HIV e de Sífilis na Gestação e no Parto no Distrito Federal", conduzida pelo Departamento Nacional de DST/Aids (DN-DST/Aids-MS).

\section{OBJETIVO}

Analisar o perfil epidemiológico e a cobertura de realização do VDRL durante a gestação e o parto, em gestantes e parturientes atendidas na rede pública de saúde do Distrito Federal.

\section{METODOLOGIA}

Adotou-se a metodologia desenvolvida entre DN-DST/Aids e o Centro de Informação da Fundação Oswaldo Cruz, financiada pelo Centers for Disease Control and Prevention Global Aids Program Brazil e o United Nations Childrens Fund (Unicef). Para a execução da pesquisa distrital, o apoio do DN-DST/ Aids foi ímpar na capacitação dos pesquisadores, preparação amostral, disponibilização dos instrumentos de pesquisa e assessoria na análise dos dados.

Quanto aos recursos, a pesquisa distrital recebeu financiamento da Fundação de Ensino e Pesquisa em Ciências da Saúde (Fepecs). Obteve-se também aprovação do comitê de ética em pesquisa da Secretaria de Estado de Saúde do Distrito Federal, potocolo $n^{\circ} 389 / 2008$.

\section{Amostra}

Considerando o estudo completo, calculou-se a amostra das participantes, a partir da estimativa de $0,41 \%$ de gestantes infectadas pelo HIV no Brasil em 2004, sendo o intervalo de confiança de $95 \%$, com erro bilateral de $0,2 \%$. Estabeleceu-se, assim, a amostra de 4 mil mulheres residentes no DF, com a seguinte representatividade nos hospitais públicos selecionados: Hospital Regional da Asa Sul (710), Hospital Regional da Asa Norte (300), Hospital Regional da Ceilândia (650), Hospital Regional de Brazilândia (140), Hospital Regional de Taguatinga (550), Hospital Regional de Sobradinho (270), Hospital Regional de Planaltina (330), Hospital Regional de Samambaia (310), Hospital Regional do Gama (450), Hospital Regional do Paranoá (200) e Hospital Universitário de Brasília (90).

\section{Instrumento de coleta de dados}

A coleta das informações foi realizada em formulário padronizado intitulado Formulário-Padrão para Preenchimento das Informações das Parturientes, contendo a seguinte estrutura: Bloco A (identificação), Bloco B (dados sociodemográficos), Bloco $C$ (dados de pré-natal), Bloco $D$ contendo variáveis diversas relativas ao parto (data do parto, situação do recém-nascido e resultado dos testes de HIV e de sífilis), à parturiente (identificação, número da declaração de nascimento, idade, grau de escolaridade, raça/cor, local de residência), ao pré-natal (fez pré-natal, tem cartão do prénatal), ao cartão do pré-natal (número de consultas, data e trimestre de início do pré-natal, resultados e datas dos testes de síflis e anti-HIV) e informações do parto. Quanto à variável sobre raça/cor, utilizou-se a classificação do Instituto Brasileiro de Geografia e Estatística (IBGE), isto é, por autoclassificação em uma constelação de cinco itens: branco, pardo, preto, amarelo e indígena. 
Para os dados secundários, utilizaram-se instrumentos de coleta: cartão de pré-natal, fichas de admissão, prontuários (variáveis sociodemográficas das gestantes e parturientes selecionadas, resultados dos testes de HIV e sífilis no pré-natal e no parto, condições de nascimento). Atinente aos dados primários, utilizou-se a entrevista como técnica de coleta de dados relativos a escolaridade e raça/cor.

Utilizou-se o Termo de Consentimento Livre e Esclarecido (TCLE), garantindo a ética na pesquisa.

\section{Trabalho de campo}

Os entrevistadores foram capacitados, previamente, para o levantamento de dados primários e secundários nas diferentes fontes e o registro no instrumento padronizado. A equipe de entrevistadores inicialmente foi composta de 19 profissionais de saúde, representantes de 11 hospitais. Entre os problemas relativos à coleta de dados, ocorreram substituições de entrevistadores e o fechamento temporário de algumas maternidades. Assim, a coleta dos dados, iniciada em junho de 2009, encerrou em maio de 2010. Totalizaram 24 entrevistadores. Para cada maternidade selecionada, houve um pesquisador responsável pela coleta das informações das parturientes.

A coordenação, supervisão, coleta, análise e divulgação dos dados foram realizadas por profissionais da SES/DF. Utilizou-se a estrutura física (salas) e os insumos da Secretaria de Estado de Saúde do Distrito Federal (SES/DF).

\section{Análise dos dados}

Para a análise estatística, utilizou-se o aplicativo SPSS, versão 13.0. A base de dados foi calibrada considerando o desenho amostral e a distribuição por região administrativa de residência dos nascidos vivos, no período do estudo, segundo o Sistema de Informações de Nascidos Vivos (Sinasc). Foram excluídas as parturientes cujo instrumento indicava que a mesma não residia no DF ou sem essa informação.

\section{RESULTADOS}

Os resultados da sorologia e os dados das demais variáveis estudadas foram obtidos no cartão da gestante e no prontuário hospitalar. Para o cálculo da prevalência, consideraram-se os resultados de VDRL reagente, independente da titulação, das parturientes que possuíam resultado do exame feito na admissão para o parto.

Ao fim do estudo, coletaram-se informações de 3.790 parturientes. Entretanto, somente 3.726 instrumentos foram analisados, pois cumpriam a exigência de as partícipes serem exclusivamente residentes no DF.

A tabela 1 apresenta a caracterização das parturientes segundo faixa etária, grau de escolaridade e raça/cor. A amostra foi composta, majoritariamente, de mulheres na faixa etária de 20 a 34 anos, correspondendo a 73\% (2.721) das entrevistadas. Destacam-se 14,4\% (536) entre 15 e 19 anos e 0,5\% (18) entre 10 e 14 anos de idade. A média etária foi 26 anos, a mediana, 26 anos, e a moda, 22 anos.

Quantoà escolaridade, predominaram mulheres $(40,4 \%)$ que possuem o ensino fundamental completo ou médio incompleto, seguidas daquelas que cursaram o ensino médio completo ou superior incompleto $(28,1 \%)$ e o elementar incompleto $(23,6 \%)$.

Ao analisar a variável raça/cor autodeclarada pelas entrevistadas, 54,7\% referiram que são pardas, $25,3 \%$, brancas, e $15,5 \%$, negras.

Tabela 1 - Distribuição das parturientes por faixa etária, grau de escolaridade e raça/cor. Distrito Federal, 2010

\begin{tabular}{|l|c|c|}
\hline Características das participantes & No & $\%$ \\
\hline Faixa etária da mãe & & \\
$10-14$ & 18 & 0,5 \\
$15-19$ & 536 & 14,4 \\
$20-24$ & 1.084 & 29,1 \\
$25-29$ & 958 & 25,7 \\
$30-34$ & 679 & 18,2 \\
$35-39$ & 336 & 9,0 \\
$40-44$ & 96 & 2,6 \\
$45-49$ & 9 & 0,2 \\
Em branco & 10 & 0,3 \\
\hline Escolaridade & & \\
Analfabeto/< de um ano de estudo & 19 & 0,5 \\
Elementar incompleto & 880 & 23,6 \\
Elem. completo e fund. incompleto & 108 & 2,9 \\
Fundamental completo e ensino & 1.504 & 40,4 \\
médio incompleto & & \\
Ensino médio completo & 1.045 & 28,1 \\
e superior incompleto & & \\
Superior completo ou mais & 128 & 3,4 \\
Em branco & 41 & 1,1 \\
Raça & & \\
Parda & 2.040 & 54,7 \\
Branca & 941 & 25,3 \\
Negra & 578 & 15,5 \\
Amarela & 34 & 0,9 \\
Indígena & 15 & 0,4 \\
Ignorada & 3.726 & 100,0 \\
\hline Total & & \\
\hline
\end{tabular}


Referente ao pré-natal, verificou-se que a cobertura considerando qualquer número de consultas foi de $97,6 \%$. No grupo que realizou o pré-natal, $89,6 \%$ das gestantes tiveram quatro ou mais consultas. Quando a mesma análise considerou sete ou mais consultas no pré-natal, a cobertura foi de $58,9 \%$. Ressalta-se que apenas $8 \%$ fizeram menos de quatro consultas. Analisando os dados sobre o início do pré-natal, observou-se que $48,9 \%$ das parturientes iniciaram no primeiro trimestre de gestação e $37,5 \%$, no segundo (tabela 2).

Este trabalho verificou que $88 \%$ das parturientes atendidas em maternidades públicas no DF realizaram pelo menos um teste de síflis (VDRL) durante a gravidez. Dessas, 37,4\% conseguiram realizar os dois testes e apenas $19,6 \%$ tiveram acesso aos três testes preconizados. Quanto ao teste de sífilis exigido no momento do parto, observou-se que $91,5 \%$ das parturientes conseguiram realizá-lo (tabela 2).

A figura 1 mostra as desigualdades na cobertura do VDRL segundo escolaridade e renda per capita do local de moradia da parturiente. Entre as mulheres analfabetas ou com menos de um ano de instrução, 35\% não fizeram nenhum teste e apenas $10 \%$ conseguiram realizar os três testes indicados.

Tabela 2 - Distribuição das parturientes de acordo com a cobertura do pré-natal e realização dos testes de sífilis (VDRL). Distrito Federal, 2010

\begin{tabular}{|l|c|c|}
\hline Situação & $N^{\circ}$ & $\%$ \\
\hline Receberam atendimento pré-natal & 3638 & $97,6 \%$ \\
Iniciaram o pré-natal no primeiro trimestre & 1779 & $48,9 \%$ \\
Fez 4 ou mais consultas de pré-natal & 3349 & $86,6 \%$ \\
Fez pelo menos 1 teste de síflis (VDRL) & 3280 & $90,1 \%$ \\
Fez 2 testes de síflis (VDRL) & 1395 & $37,4 \%$ \\
Fez 3 testes de sífilis (VDRL) & 730 & $19,6 \%$ \\
Teste de síflis no parto (VDRL) & 3412 & $91,5 \%$ \\
\hline
\end{tabular}

Esse cenário é igualmente preocupante entre as gestantes que não completaram o ensino fundamental, pois a cobertura dos três testes foi de $15,8 \%$, enquanto a cobertura entre as mulheres que concluíram o ensino superior foi de $30,5 \%$.

Destaca-se que, quanto maior a renda per capita do local de moradia, maior a proporção de gestantes que conseguiram realizar os três testes de sífilis preconizados. Nos locais com renda superior a três salários mínimos, 30,2\% das parturientes conseguiram fazer os três testes, $37,7 \%$ conseguiram realizar os dois testes de sífilis, $25,3 \%$ fizeram apenas um teste e $6,8 \%$ não conseguiram realizar nenhum teste ou não tinham essa informação no cartão de pré-natal. Em contraponto, entre as gestantes residentes nas localidades com renda per capita

Figura 1 - Cobertura do teste de sífilis (VDRL) no pré-natal segundo escolaridade e renda per capita de residência. Distrito Federal, 2010
Cobertura do teste de sífilis segundo escolaridade. Distrito Federal, 2010

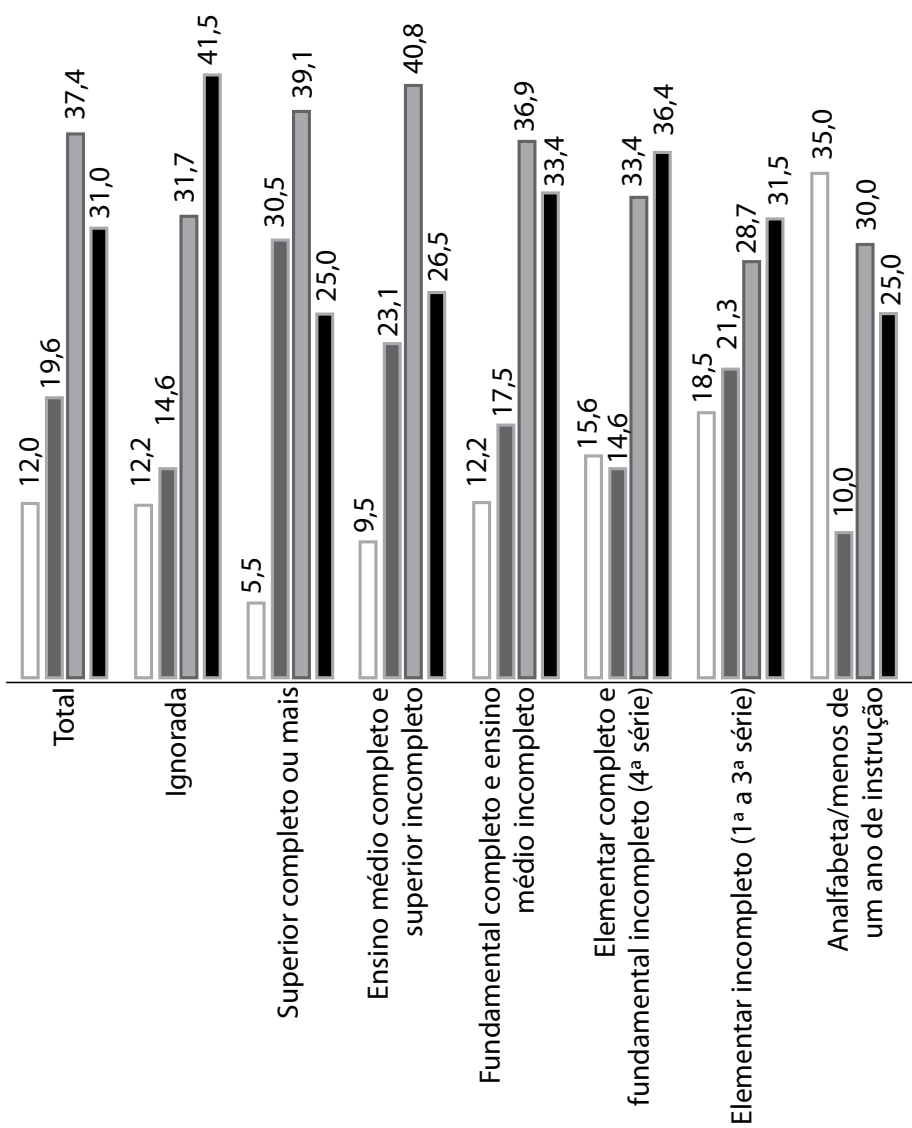

Cobertura do teste de sífilis segundo renda per capita de residência. Distrito Federal, 2010

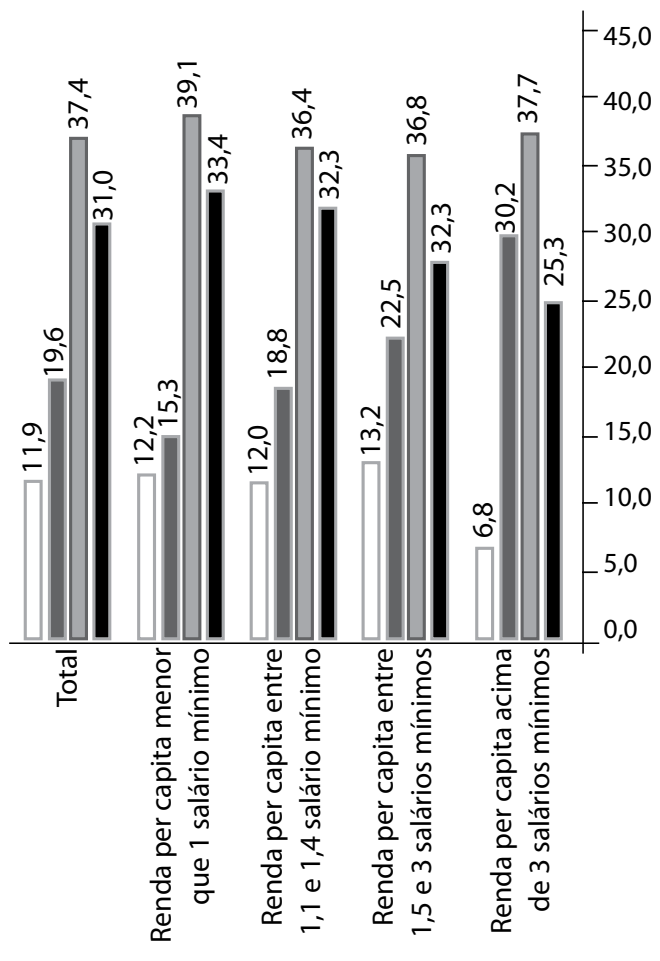


Figura 2 - Cobertura do teste de sífilis (VDRL) no pré-natal segundo faixa etária e raça/cor. Distrito Federal, 2010

Cobertura do teste de sífilis segundo faixa etária.

Distrito Federal, 2010

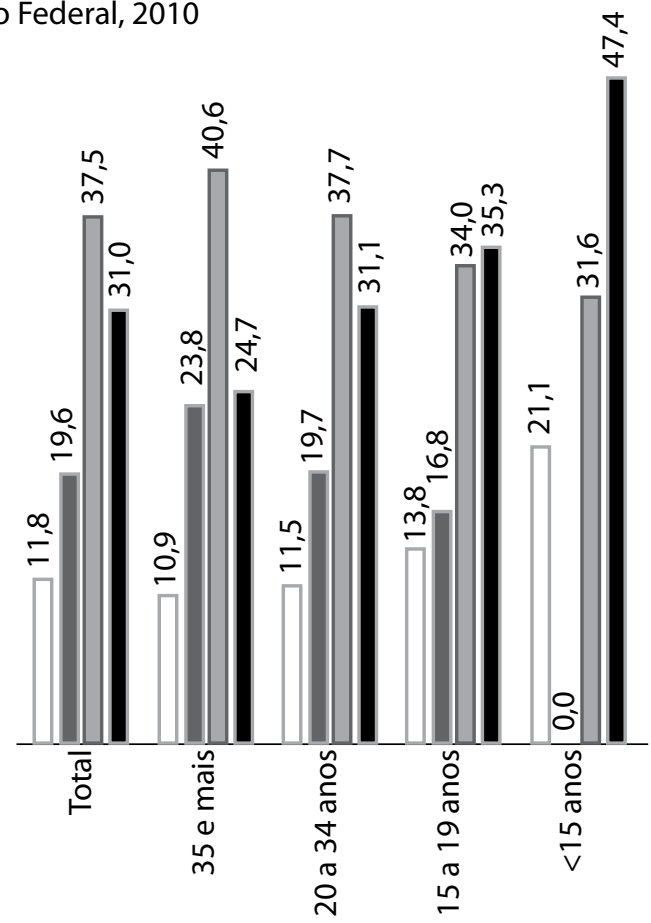

Cobertura do teste de sífilis segundo raça/cor.

Distrito Federal, 2010

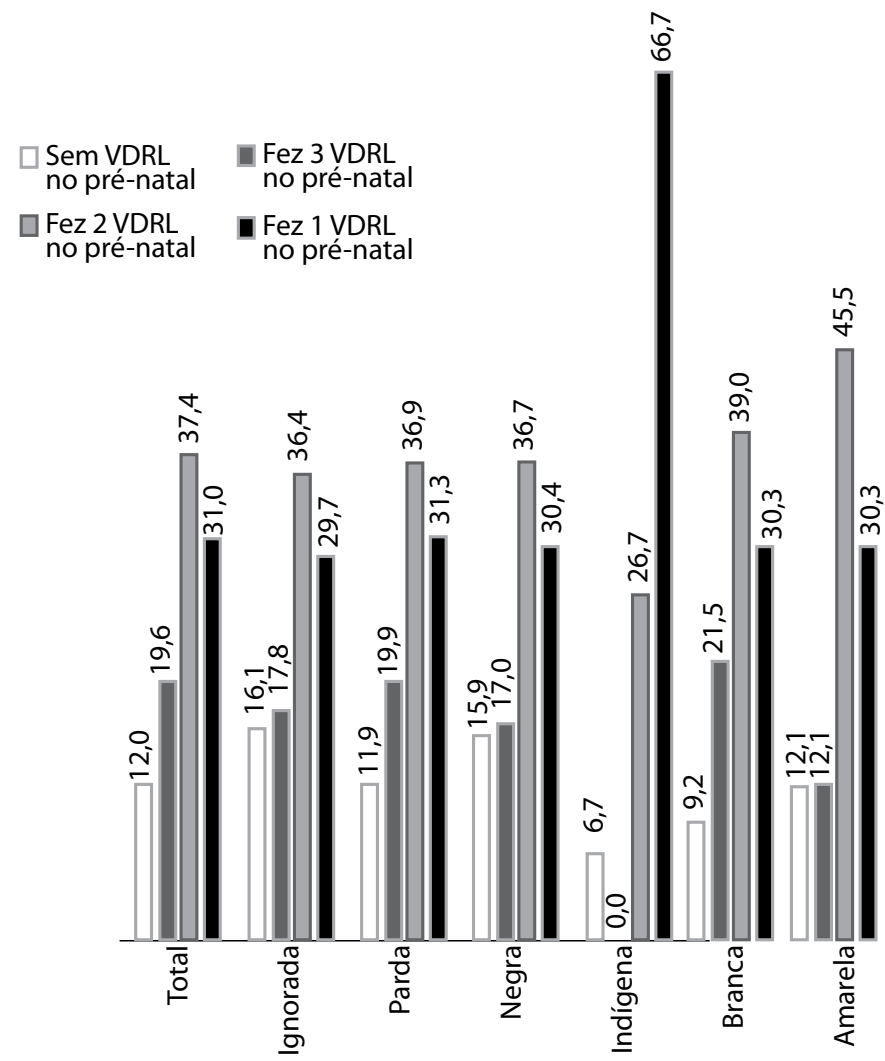

menor do que um salário mínimo, esse quadro foi diferente: apenas $15,3 \%$ conseguiram realizar os três testes exigidos e a proporção de quem não fez nenhum teste foi de $12,2 \%$.

Comparando a cobertura do teste de sífilis durante a gravidez por faixa etária, observou-se um declínio na cobertura dos três testes preconizados, variando de $23,8 \%$ entre as mulheres com 35 anos e mais a $0 \%$ nas gestantes com menos de 15 anos. Ao analisar a cobertura do teste de sífilis no pré-natal (figura 2) baseada na variável raça/cor, verificaram-se resultados de importância epidemiológica. Realizaram pelo menos dois testes VDRL $65,5 \%$ das mulheres brancas, $56,8 \%$ das mulheres pardas, $53,7 \%$ das mulheres negras, 57,6\% nas de raça amarela, e um resultado bastante preocupante entre as mulheres de raça indígena, ou seja, apenas $26,7 \%$ realizaram pelo menos dois testes.

Das $3.408(91,5 \%)$ parturientes que realizaram VDRL no momento da admissão para o parto, 20 apresentaram VDRL reagente, o que representa uma taxa de prevalência de $0,59 \%$ (IC 95\%: 0,38\%-0,91\%).

\section{DISCUSSÃO}

A principal limitação do estudo relaciona-se à qualidade das informações de algumas variáveis. Trata-se de um problema que ocorre em estudos de levantamento de dados secundários, pois geralmente são coletados por profissionais não envolvidos na pesquisa. Neste estudo, observou-se

Tabela 3 - Prevalência de VDRL reagente, no momento da admissão para o parto, entre as parturientes da rede pública de saúde do Distrito Federal, 2010

\begin{tabular}{|l|c|c|c|}
\hline Resultado & $\mathbf{N}$ & Prevalência (\%) & IC95\% \\
\hline Com VDRL reagente & 20 & 0,59 & $(0,38-0,91)$ \\
Com VDRL não reagente & 3.388 & 99,41 & \\
Total de examinadas & 3.408 & 100,00 & - \\
\hline
\end{tabular}

limitação no preenchimento correto da fonte de dados - o cartão da gestante -, pois, se a maioria das parturientes estudadas fez pré-natal e portava o cartão, identificaram-se problemas de completitude dos dados laboratoriais. Ademais, o resultado do VDRL no momento do parto, que, por norma nacional, deve ser oferecido à parturiente, não foi encontrado em alguns prontuários. Tal constatação indica que o exame não foi realizado - por falha dos serviços de saúde em realizálo ou por recusa da parturiente.

A despeito das dificuldades apontadas, a análise de achados clínicos e laboratoriais permitiu a confirmação de variáveis associadas à dificuldade de acesso ao teste diagnóstico de sífilis no pré-natal, como demonstrado em outros estudos epidemiológicos.

Para a análise de cobertura dos testes HIV e sífilis, utilizou-se o conceito de "cobertura efetiva" de Tanahashi ${ }^{(8)}$, que mensura a interação serviço-pessoas destinatárias da mesma. A cobertura 
dos testes durante a gravidez corresponde à proporção de parturientes portadoras do cartão de pré-natal na hora do parto, que fizeram pré-natal e cujos resultados dos testes estavam registrados no cartão antes do nascimento do filho ${ }^{(9)}$.

A alta cobertura de pré-natal $(89,9 \%$ com quatro ou mais consultas) observada em parturientes no Distrito Federal contrasta com o baixo cumprimento das diretrizes estabelecidas na portaria no $37 / 2008$ para reduzir a sífilis congênita e a transmissão vertical do HIV, referentes à testagem para sífilis durante a gravidez. Ressalta-se que a proporção de gestantes que realizaram sete ou mais consultas $(58,5 \%)$ se encontra abaixo da meta prevista no Pacto pela Vida 2010/2011, que é de 65,25\% para o DF ${ }^{(10)}$.

Este estudo observou que, durante o pré-natal, $90,2 \%$ das gestantes obtiveram pelo menos um resultado do teste de sífilis (VDRL), sendo que apenas 20,1\% obtiveram três resultados anotados no cartão, indicando possíveis falhas no atendimento às gestantes.

Aanálise dasinformaçõesapresentadas neste estudo corrobora com os dados nacionais publicados, revelando que os fatores socioeconômicos impedem a eliminação da sífilis congênita(11-14). Os achados confirmam a influência do nível de escolaridade e da renda per capita do local de moradia no acesso ao diagnóstico de sífilis no Distrito Federal. Quanto menor a escolaridade e a renda per capita da gestante, maior a proporção dessas mulheres sem VDRL registrado no cartão de pré-natal. Tal achado sinaliza problemas relacionados ao acesso aos serviços de saúde, aderência ao tratamento e vulnerabilidade social.

Outra condição a destacar refere-se à idade das gestantes, sendo grande a proporção de adolescentes menores de 15 anos $(21,1 \%)$, sem acesso ao teste de sífilis durante a gravidez. Esse é um resultado inquietante, pois, em pesquisa realizada em âmbito nacional com puérperas, a idade menor do que 14 anos na primeira gravidez estava fortemente associada à positividade do VDRL ${ }^{(13)}$. A sífilis na adolescência mostra-se como importante marcador de vulnerabilidade às doenças sexualmente transmissíveis, exigindo que os serviços de saúde repensem ações de prevenção à infecção pelo HIV/ Aids e outras DST para esse segmento populacional ${ }^{(13,14)}$.

$\mathrm{Na}$ análise do acesso aos testes segundo raça/cor, observou-se a situação desfavorável das mulheres indígenas em relação às brancas, pretas e pardas, pois a maior proporção das parturientes que se declararam indígenas $(66,7 \%)$ tinha apenas o registro de um exame de VDRL no cartão de pré-natal.

A prevalência da sífilis gestacional encontrada nas maternidades públicas distritais $(0,59 \%)$ preenche um dos critérios de eliminação da sífilis congênita, estabelecidos pela OMS - ou seja, a prevalência do agravo durante a gravidez menor que $1 \%{ }^{(15)}$. No entanto, o outro critério estabelecido pela OMS refere que $95 \%$ das grávidas infectadas sejam tratadas adequadamente. Notou-se que esse critério não está sendo atingido, devido à taxa de incidência de sífilis congênita no DF, que em 2010 subiu para 2,1 casos por mil nascidos vivos ${ }^{(1)}$, indicando possíveis falhas na assistência à gestante.

O percentual de parturientes sem informação quanto ao resultado da sorologia para o VDRL no momento do parto foi baixo e distribuído nas diversas maternidades estudadas.

\section{CONCLUSÕES}

Este trabalho proporcionou parâmetros da rede pública de saúde local para o monitoramento das ações referentes à prevenção da transmissão vertical da sífilis. A prevalência de sífilis foi aproximadamente metade da estimada em parturientes para o Brasil, por estudos do Ministério da Saúde, que também as mensurou por meio de resultados de VDRL ${ }^{(16)}$. Esse dado contrasta com a alta prevalência de sífilis congênita registrada no Distrito Federal conforme os dados de notificação compulsória ${ }^{(1)}$. Novos estudos devem verificar os fatores relacionados ao tratamento adequado e oportuno das gestantes durante o pré-natal, pois falhas de tratamento podem ser responsáveis pelo elevado número de casos de sífilis congênita.

Esta pesquisa possibilitou conhecer questões relativas à qualidade do prénatal e identificar falhas nas ações de prevenção e controle da transmissão vertical da sífilis na rede pública de saúde do Distrito Federal, que precisam ser corrigidas.

Melhorar os resultados desses indicadores e, por consequência, a qualidade dos serviços prestados à população requer nova forma de conceber o sistema de saúde, que abrange a transformação das relações de trabalho da equipe de saúde; o repensar da prestação de um serviço pura e simplesmente entendido como o cumprimento da realização de atos formais e burocráticos; e uma nova forma de olhar o paciente, de escuta do outro, de disponibilidade e responsabilização, visando à integralidade do cuidado ${ }^{(17)}$.

O alcance dessa integralidade é um desafio que envolve todos os atores implicados, tanto na formulação de políticas quanto na construção do conhecimento e implementação das práticas de saúde.

Os próximos passos propostos pelo grupo de trabalho da gerência de DST/Aids/hepatites virais são: aprofundar a análise dos dados em parceria com o Ministério da Saúde (estimativas de prevalências); realizar reuniões regionais com gestores e profissionais envolvidos na atenção à saúde materno-infantil para discussão dos resultados e elaboração de propostas de trabalho; realizar este estudo com uma 
periodicidade bianual para monitoramento da portaria $\mathrm{n}^{\circ}$ $37 / 2008$, com a inclusão dos marcadores de hepatites virais.

\section{Agradecimentos}

Pesquisa desenvolvida com recursos da Fundação de Ensino e Pesquisa em Ciências da Saúde. Grupo de Estudos na área da
Saúde e Grupo de Epidemiologia. Entrevistadores da pesquisa: Emilio Ribeiro, Talita Cunha, Maria Lúcia Cardoso, Gilda da Silva, Edna Flor, Rosana Pessoa, Edilmar Lucena, Jucinei Batista, Maria do Socorro Rangel, Ivone Santos, Ivete Sevilla, Kelly Alves, Elisete Carvalho, Josele Ferreira, Aline Cabral, Ana Karla Silva, Karla Queiroz, Leda Nunes, Hygor Elias.

\section{Referências}

1. Secretaria de Estado de Saúde (DF). Subsecretaria de Vigilância em Saúde. Diretoria de Vigilância Epidemiológica. Gerência de DST/Aids e HV. Bol Epidemiol DST/Aids Distr Fed. 2011(1).

2. Ministério da Saúde (BR). Secretaria de Políticas de Saúde. Coordenação Nacional de DST e Aids. Projeto de eliminação da sífilis congênita. Manual de assistência e vigilância epidemiológica. Brasília: Ministério da Saúde; 1998. 3. Ministério da Saúde (BR). Protocolo para a prevenção de transmissão vertical de HIV e sífilis: manual de bolso. Brasília: Ministério da Saúde; 2007. 4. Ministério da Saúde (BR). Secretaria de Vigilância em Saúde. Programa Nacional de DST E Aids. Plano operacional para redução da transmissão vertical do HIV e da sífilis. Brasília: Ministério da Saúde; 2007.

5. Governo (DF). Secretaria de Estado de Saúde. Portaria n 37 de 4 de abril de 2008. Normatiza ações pró-redução da transmissão vertical do HIV e sífilis. Diário Oficial do Distrito Federal, Poder Executivo, Brasília, DF, 8 abr. 2008. Seção 1, p. 11-6.

6. Ministério da Saúde (BR). Secretaria Executiva. Departamento de Apoio à Descentralização. Coordenação-Geral de Apoio à Gestão Descentralizada. Diretrizes operacionais dos pactos pela vida, em defesa do SUS e de gestão. $2^{\text {a }}$ ed. Brasília: Ministério da Saúde; 2006. Série A. Normas e Manuais Técnicos. 7. Arruda DCB, Silva O, Tavares LHDC. Experiência de pactuação pró-redução da transmissão vertical do HIV e da sífilis no Distrito Federal. Bol Epidemiol SDT/Aids Distr Fed. 2008;7(1):6-7.

8. Tanahashi T. Health services coverage and its evaluation. Bull World Health Organ. 1978;78:1246-55.

9. Szwarcwald CL. HIV testing during pregnancy: use of secondary data to estimate 2006 test coverage and prevalence in Brazil [Internet, citado em 2010 ago 04]. Disponível em: http://www.scielo.br/scielo.php?script=sci arttext\&pid=S1413-86702008000300002\&lng=en\&nrm=iso.

10. Ministério da Saúde (BR). Portaria № 2669, de 3 de novembro de 2009.
Estabelece as prioridades, objetivos, metas e indicadores de monitoramento e avaliação do Pacto pela Saúde, nos componentes pela Vida e de Gestão, e as orientações, prazos e diretrizes do seu processo de pactuação para o biênio 2010-2011 [Internet, citado em 2010 ago 04]. Disponível em: http://bvsms. saude.gov.br/bvs/saudelegis/gm/2009/prt2669_03_11_2009.html.

11. Ramos Jr AN, Matida LH, Saraceni V, Veras MASM, Pontes RJS. Control of mother-to-child transmission of infectious diseases in Brazil: progress in HIV/ Aids and failure in congenital syphilis. Cad Saúde Pública. 2007;23(Suppl 3):S370-8.

12. Lago EG, Rodrigues LC, Fiori RM, Stein AT. Congenital syphilis: idenfication of two distinc profiles os maternal characteristics associated with risk. Sex Transm Dis. 2004;31(1):33-7.

13. Rodrigues CS, Guimarães MDC. Positividade para sífilis em puérperas: ainda um desafio para o Brasil [Internet, citado em 2011 dez 19]. Disponível em: http://www.scielosp.org/scielo.php?script=sci_arttext\&pid=S102049892004000900003\&lng=en. http://dx.doi.org/10.1590/S102049892004000900003.

14. Leal MC, Gama SGN, Cunha CB. Desigualdades raciais, sociodemográficas e na assistência ao pré-natal e ao parto, 1991-2001. Rev Saúde Pública. 2005;39(1):100-7.

15. Valderrama J, Bautista MAU. Sífilis materna y sífilis congénita: definiciones de caso [Internet, citado em 2011 dez 05]. Disponível em: http://www.paho. org/spanish/dd/ais/be_v26n1-sp-sifilis.htm.

16. Szwarcwald CL, Barbosa Junior A, Miranda AE, Paz LC. Resultados do estudo sentinela-parturiente, 2006: desafios para o controle da sífilis congênita no Brasi. DST - J Bras Doenças Sex Transm. 2007;19(3-4):128-33. 17. Campos CEA. O desafio da integralidade segundo as perspectivas da vigilância da saúde e da família saúde da família. Ciênc Saúde Coletiva. 2003;8(2):569-84. 